\title{
Targeting cancer stem cells in glioblastoma multiforme using $m$ TOR inhibitors and the differentiating agent all-trans retinoic acid
}

\author{
MARISSA D. FRIEDMAN*, DHRUVE S. JEEVAN*, \\ MICHAEL TOBIAS, RAJ MURALI and MEENA JHANWAR-UNIYAL \\ Department of Neurosurgery, New York Medical College, Valhalla, NY 10595, USA
}

Received April 18, 2013; Accepted May 21, 2013

DOI: $10.3892 / o r .2013 .2625$

\begin{abstract}
Glioblastoma multiforme (GBM), the most aggressive primary brain tumor, portends a poor prognosis despite current treatment modalities. Recurrence of tumor growth is attributed to the presence of treatment-resistant cancer stem cells (CSCs). The targeting of these CSCs is therefore essential in the treatment of this disease. Mechanistic target of rapamycin (mTOR) forms two multiprotein complexes, mTORC1 and mTORC2, which regulate proliferation and migration, respectively. Aberrant function of mTOR has been shown to be present in GBM CSCs. All-trans retinoic acid (ATRA), a derivative of retinol, causes differentiation of CSCs as well as normal neural progenitor cells. The purpose of this investigation was to delineate the role of mTOR in CSC maintenance, and to establish the mechanism of targeting GBM CSCs using differentiating agents along with inhibitors of the mTOR pathways. The results demonstrated that ATRA caused differentiation of CSCs, as demonstrated by the loss of the stem cell marker Nestin. These observations were confirmed by western blotting, which demonstrated a time-dependent decrease in Nestin expression following ATRA treatment. This effect occurred despite combination with mTOR (rapamycin), PI3K (LY294002) and MEK1/2 (U0126) inhibitors. Expression of activated extracellular signal-regulated kinase $1 / 2(\mathrm{pERK} 1 / 2)$ was enhanced following treatment with ATRA, independent of mTOR pathway inhibitors. Proliferation of CSCs, determined by neurosphere diameter, was decreased following treatment with ATRA alone and in combination with rapamycin. The
\end{abstract}

Correspondence to: Dr Meena Jhanwar-Uniyal, Department of Neurosurgery, New York Medical College, Valhalla, NY 10595, USA

E-mail: meena_jhanwar@nymc.edu

${ }^{*}$ Contributed equally

Key words: mTOR, glioblastoma, all-trans-retinoic acid, cancer stem cells motility of GBM cells was mitigated by treatment with ATRA, rapamycin and LY29002 alone. However, combination treatment augmented the inhibitory effect on migration suggesting synergism. These findings indicate that ATRAinduced differentiation is mediated via the ERK1/2 pathway, and underscores the significance of including differentiating agents along with inhibitors of mTOR pathways in the treatment of GBM.

\section{Introduction}

Glioblastoma multiforme (GBM), a grade IV astrocytoma, is the most frequent and aggressive primary brain tumor. Despite the increased understanding of the oncological mechanisms underlying GBM pathophysiology, and treatment advances, only a marginal improvement in overall survival has been seen during the first decade of the 21st century (1). Abnormalities in multiple genetic pathways have been identified including amplification of epidermal growth factor (EGFR), loss of chromosome 10q, mutation of phosphatase and tensin homolog (PTEN), mutation of p53, and concomitant loss of p16 and p18 (2-4). Investigation into similarities between growth factor signaling elements implicated in GBM progression, and those that control crucial stages in neural development has provided evidence signifying neural stem and/or progenitor cells as the cell type of origin for GBM (5-7). These cancer stem cells (CSCs) carry three distinct properties: self-renewal, ability to differentiate into multiple lineages and extensive proliferation. Their presence in tumors has been demonstrated through the identification of specific antigenic markers, and use of co-culture conditions that were originally developed for normal neural stem cells $(6,8,9)$.

Aberrant signaling pathways contributing to abnormal cell migration, invasion, proliferation, as well as CSC maintenance are responsible for the aggressive nature of GBM (10). Many of the identified mutations result in activation of the lipid kinase PI3K and its downstream target, the plekstrin-homologydomain serine threonine kinase AKT. AKT has over 40 downstream targets (11). Prominent among these is mechanistic target of rapamycin (mTOR; AKA mammalian target of rapamycin) and recent reports have linked PI3K/Akt/mTOR activity directly to CSC expansion and maintenance $(10,12)$. 
mTOR is an atypical serine/threonine kinase that regulates several processes including autophagy, ribosome biogenesis, and metabolism by integrating signals from growth factors, nutrients, oxygen and energy status (13). Recent studies have indentified two structurally and functionally distinct mTOR-containing multiprotein complexes, mTOR complex 1 (mTORC1) and complex 2 (mTORC2). The two complexes consist of unique mTOR-interacting proteins, which determine their substrate specificity. mTORC1 acts as a downstream effector of PI3K/Akt signaling, promoting cell growth, proliferation, and CAP-dependent protein translation, alongside inhibiting autophagy. mTOR2 phosphorylates AKT at Ser473, and as an upstream activator regulates serum/glucocorticoid regulated kinase (SGK) as well as PKC. mTORC2 also modifies cytoskeletal elements and as a result is involved in controlling cellular migration. mTOR has been implicated in promoting the maintenance of stem cells and CSCs indicating its inhibition as an approach to prevent self-renewal of stem cells $(10,12,14)$.

An alternate proposed mechanism to target the CSCs of GBM is to promote differentiation and therefore increase sensitivity to therapy. All-trans retinoic acid (ATRA), a derivative of retinoid, is capable of differentiating a variety of stem cells, as well as normal neural progenitor cells (15). Retinoic acid and its derivatives activate retinoic acid receptors and retinoid $\mathrm{X}$ receptor (RAR-RXR) complexes and induce differentiation of neural stem cells (16). A combination of ATRA and interferon $\gamma$ (IFN $\gamma$ ) has shown the ability to induce both differentiation and apoptosis of GBM cells independent of their PTEN status (17-19), and clinically the use of ATRA with cytosine arabinoside resulted in stability of recurrent GBM (20).

This investigation addresses means to target GBM CSCs using the combination of differentiating agent ATRA with inhibitors of the PI3K/AKT/mTOR pathways, and thus provide a novel therapeutic approach.

\section{Materials and methods}

Cell line and reagents. The human glioblastoma cell lines U87 and LN18 were used. These are commercially available neurosphere forming primary GBM cell lines (ATCC, Manassas, VA, USA). U87 (21) cell line exhibits p16INK loss, PTEN loss and wild-type p53. LN18 (22) cell line exhibits intact PTEN and mutant p53 at codon 238. Cells were maintained in Dulbecco's modified Eagle's medium (DMED; Invitrogen, Carlsbad, CA, USA) supplemented with $10 \%$ FBS and $1 \%$ penicillin/streptomycin/amphotericin in a humidified incubator with $5 \% \mathrm{CO}_{2}$ at $37^{\circ} \mathrm{C}$. Cells were made quiescent by serum deprivation $24 \mathrm{~h}$ prior to treatment. Treatment included the mTORC1 inhibitor rapamycin (RAPA, $100 \mathrm{nM}$; EMD Chemicals, Gibbstown, NJ, USA), PI3K inhibitor LY294002 (LY, $10 \mu \mathrm{M}$; EMD Chemicals), MAP kinase 1 and 2 (MEK1/2) inhibitor U0126 (10 $\mu \mathrm{M}$; Sigma-Aldrich, St. Louis, MO, USA), and all-trans retinoic acid (ATRA, $10 \mu \mathrm{M}$; Sigma-Aldrich). All reagents were attained from Sigma-Aldrich.

Isolation of protein. Protein extraction was performed with whole cell lysis buffer containing $1 \%$ Triton X-100, $10 \mathrm{mM}$ Tris-HCl, $\mathrm{pH} 7.5,150 \mathrm{mM} \mathrm{NaCl}$ and $5 \mathrm{mM}$ EDTA containing phosphatase and protease inhibitors (Sigma-Aldrich). Protein concentrations were determined by the modified Lowry method (Bio-Rad Laboratories, Hercules, CA, USA).

Western blot analysis. Equal amounts of protein were resolved on a $10 \%$ SDS-PAGE gel and then electrotransferred onto nitrocellulose membrane. Membranes were processed according to the manufacturer's instructions (Santa Cruz Biotechnology, Santa Cruz, CA, USA; Cell Signaling Technology, Danvers, MA, USA). A routine procedure utilized primary antibodies for Nestin and activated extracellular regulated kinase 1 and 2 (ERK, pERK1/2 $2^{\mathrm{Th} 202 / \mathrm{Ty} 204}$ ), at 1:1000 dilutions (Santa Cruz Biotechnology), followed by detection by chemiluminescence (Millipore, Billerica, MA, USA). Blots were stripped (Pierce Protein Biology Products, Rockford, IL, USA) and re-probed with actin or respective total antibodies to ensure equal loading. Densiometric analysis was performed using ImageJ (NIH, Bethesda, MD, USA). Experiments were conducted at least three times.

Fluorescence immunohistochemistry. Cells were treated with ATRA or RAPA for $24 \mathrm{~h}$. After treatments cells were fixed in 4\% PFA, blocked with $10 \%$ goat serum in PBS/0.1\% Triton X-100, and stained with Nestin (1:500; Santa Cruz Biotechnology) according to the manufacturer's instructions. FITC-Green or Rhodamine-Red secondary was used (Jackson ImmunoResearch, West Grove, PA, USA) with 4',6-diamidino2-phenylindole (DAPI) nuclear counterstaining. Cells were visualized using imaging system of Zeiss Axiovert 100M microscope (Thornwood, NY, USA).

Neurosphere assays. Serum-starved neurospheres were treated with RAPA, LY, or ATRA for up to 3 days. Serial images of treated cells were taken at x10 using an Axiovert 100M (Zeiss) where field coordinates were maintained. Neurosphere diameters were measured (AxioVision software; Zeiss) across all visibly identifiable neurospheres by a single blinded observer, while diameters across ellipsoid neurospheres were measured diagonally across all treatments to ensure uniformity. Neurosphere diameters were normalized to day zero for each treatment. Effects of combination therapy were also assessed with ATRA and RAPA and LY.

Scratch/wound healing migration assay. The scratch wound migration technique was used to determine the motility of GBM cells following treatment. LN18 cells were grown to confluent monolayer, and when approaching $100 \%$ cell confluence scratching the surface as uniformly as possible with a pipette tip formed a wound. This initial wounding and the movement of the cells in the scratched area were photographically monitored using the Axiovert Zeiss 200 microscope (Carl Zeiss) with x10 magnification (NA 0.25). The migration rate was expressed as a percentage of the control, and it was calculated as the proportion of the mean distance between both borderlines caused by scratching, to the distance that remained cell-free after migration. Two independent series of experiments were performed in quadruplicate.

Statistical analysis. Values are presented as the means. One-way ANOVA was carried out for multiple comparisons 


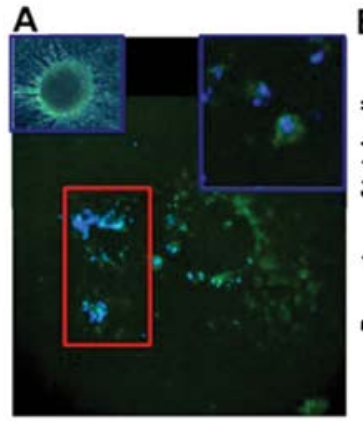

B
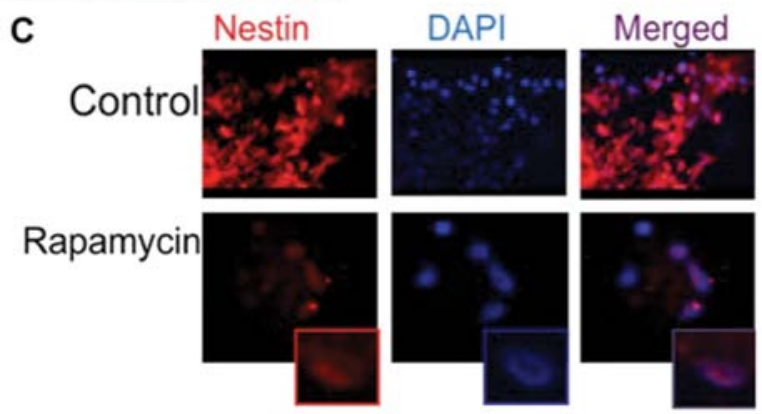

Figure 1. Immunofluorescence studies of Nestin expression. (A) Immunofluorescence analysis showing neural stem cell marker Nestin with FITC (green) staining, and DAPI nuclear staining (blue) and after treatment with all-trans retinoic acid (ATRA). The cells with stem cell characteristics express Nestin in the cytoplasm as evident by the magnified insert. The area outlined in red shows the differentiated cells that are void of Nestin expression. (B) Histogram depicting the percentage of total cells expressing Nestin (orange bar) compared to the percentage of cells no longer expressing Nestin after ATRA treatment (purple bar). (C) Immunofluorescence analysis showing DAPI nuclear staining (blue) and stem cell marker, Nestin, (red) before and after treatment with mTOR inhibitor, rapamycin (RAPA). Nestin expression was more pronounced in the nucleus following treatment with RAPA, as shown by the presence of both stains in the merged image, whereas in the control, the Nestin remained localized in the cytoplasm.

and two-tailed t-tests were used for single comparisons. A $\mathrm{P}$-value of $<0.05$ was considered significant.

\section{Results}

ATRA induces differentiation of CSCs, evidenced by depletion of stem cell marker Nestin. In order to establish that ATRA induced differentiation of CSCs, stem cells were treated with ATRA $(10 \mu \mathrm{M})$ for $24 \mathrm{~h}$ followed by immunofluorescence analysis of Nestin expression (Fig. 1A). A quantified comparison of cells expressing Nestin was performed after counterstaining with DAPI. All non-treated CSCs demonstrated Nestin expression, where only $40 \%$ of the cells expressed Nestin following treatment with ATRA (Fig. 1B). This indicates that treatment with ATRA was able to cause a significant degree of differentiation among CSCs.

mTOR inhibition alters cellular localization of Nestin. Cells treated with the mTORC1 inhibitor, RAPA $(100 \mathrm{nM})$ for $24 \mathrm{~h}$ were subjected to immunofluorescence analysis (Fig. 1C). The CSCs expressed Nestin ubiquitously and was found primarily in the cytoplasm, as evident by the absence of Nestin co-localization with DAPI in the merged image (Fig. 1C, upper panel). Following treatment with RAPA cytoplasmic expression of Nestin was lost, though strong nuclear expression was evident (Fig. 1C, lower panel).
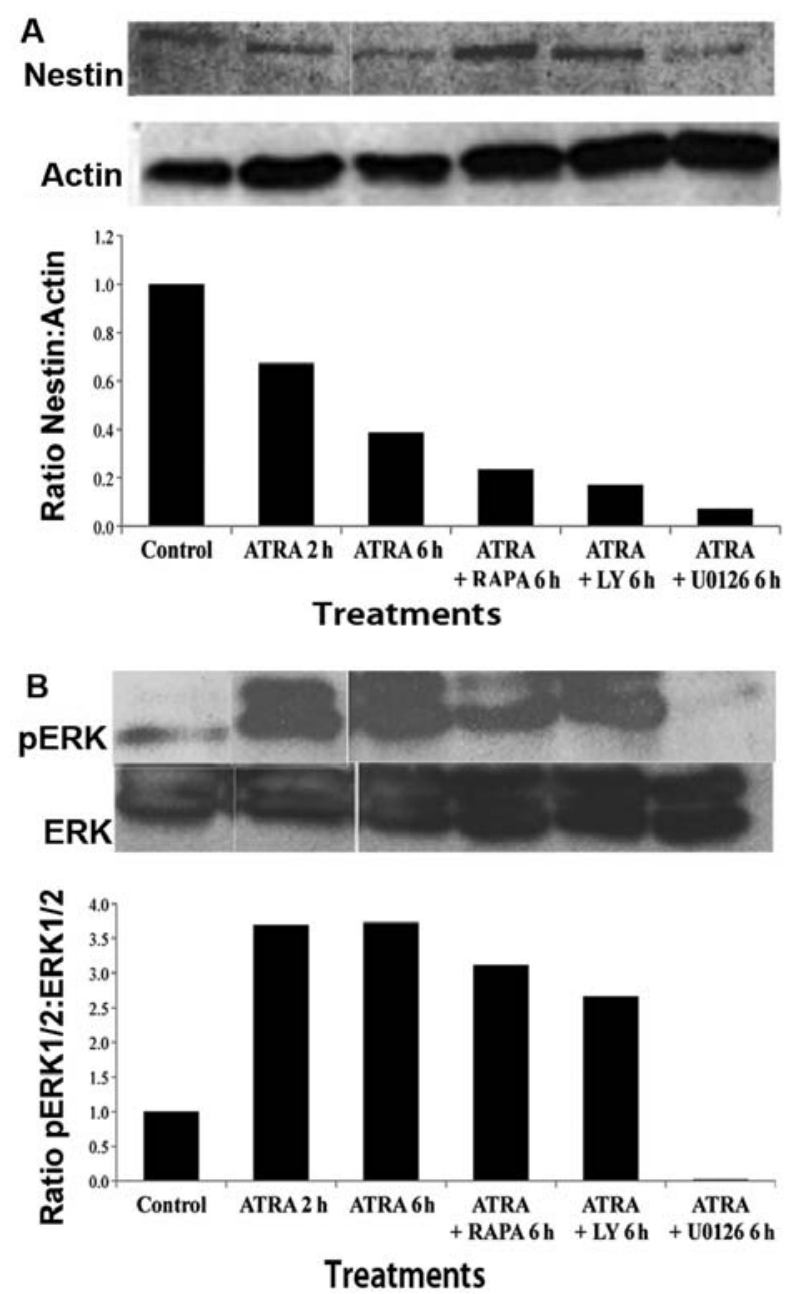

Figure 2. Western blot analysis of stem cell marker Nestin and activated ERK (A) Western blot analysis and densitometry analysis represented as Nestin to actin ratio, shows a time-dependent decrease in Nestin expression after treatment with ATRA at 2 and $6 \mathrm{~h}$. Combination treatment with ATRA and PI3K inhibitor, LY, or mTOR inhibitor RAPA, or MEK1/2 inhibitor U0126, showed a decrease in Nestin compared to control. (B) Western blot analysis and densitometry analysis, expressed as a ratio of pERK1/2 to ERK1/2, shows a time-dependent increase in extracellular-related kinase (ERK) following treatment with ATRA at 2 and $6 \mathrm{~h}$. Cells treated with ATRA in combination with LY or RAPA demonstrated a slight increase in ERK1/2 expression as compared to control. However, treatment with ATRA and MEK1/2 inhibitor, U0126, showed no increase in ERK expression, suggesting that U0126 was able to abrogate the effect of ATRA on cell differentiation.

Alterations in Nestin and ERK expression following treatment with ATRA and PI3K/mTOR inhibitors. For further analysis of the effect of ATRA on differentiation, CSCs were treated with ATRA $(10 \mu \mathrm{M})$ for either 2 or $6 \mathrm{~h}$ and western blot analysis with densitometry analysis was performed. A significant decrease in Nestin was seen after ATRA treatment as compared to control. This reduction in Nestin expression was greater at 6 than at $2 \mathrm{~h}$ suggesting a time-dependent effect. Nestin expression was examined in CSCs treated with combinations of ATRA and LY $(10 \mu \mathrm{M})$, RAPA, (100 nM) or U0126 $(10 \mu \mathrm{M})$ for $6 \mathrm{~h}$. The results illustrated a decrease in Nestin expression comparable to that of ATRA alone (Fig. 2A). The bar graph represents the arbitrary value of Nestin in comparison to actin, and demonstrates further Nestin inhibition with the use of pathway inhibitors. 

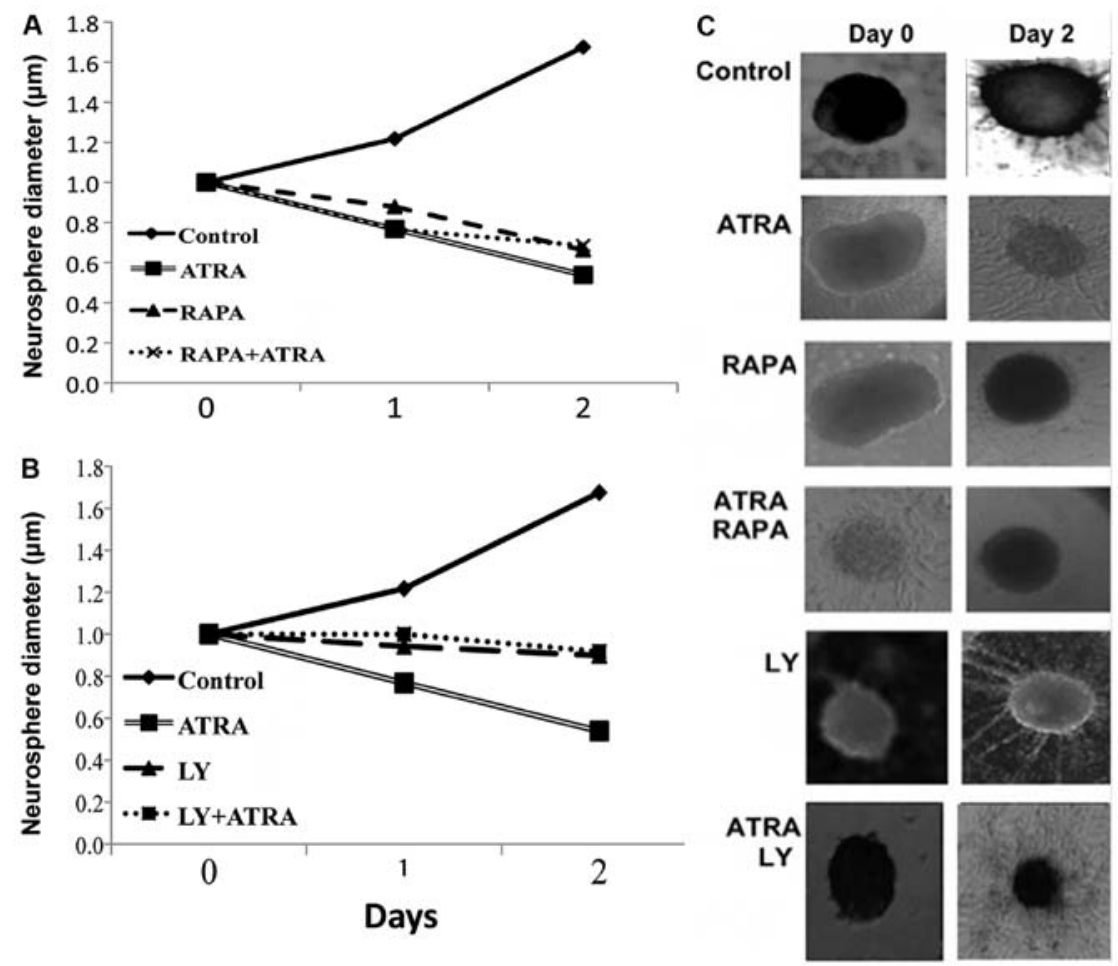

Figure 3. Stem cell proliferation assays. (A) CSC proliferation determined by using neurosphere diameter measurement at 1,2,3 and 4 days. Cells treated with RAPA, ATRA, or a combination of the two all showed a decrease in cellular proliferation. (B) CSC proliferation determined by using neurosphere diameter measurement at 1, 2, 3 and 4 days. Cells treated with LY, ATRA, or a combination of the two all showed a decrease in cellular proliferation. (C) Photographic representation of neurospheres following $48 \mathrm{~h}$ of pharmacological treatment.

In order to investigate the mechanism of differentiation induced by ATRA, we examined activated ERK1/2 (pERK1/2) expression in CSCs following ATRA treatment in the presence or absence of mTOR/PI3K/MAPK inhibitors (Fig. 2B). pERK1/2 expression was found to be increased following treatment with ATRA at 2 and $6 \mathrm{~h}$ in a time-dependent manner. pERK1/2 expression was also found to be increased following treatment with LY and RAPA in combination with ATRA. Treatment with MEK1/2 inhibitor, U0126 $(10 \mu \mathrm{M})$, in combination with ATRA resulted in no alteration in ERK1/2 expression, suggesting that U0126 was able to abrogate the activation caused by ATRA.

The effects of PI3K/mTOR inhibition in combination with differentiating agents on CSC proliferation. The proliferation of CSCs was assessed by a neurosphere assay determining the size changes in neurospheres. As expected with time there was an exponential increase in cell proliferation in controls (Fig. 3). Following treatment with ATRA, and in the presence of mTOR or PI3K inhibitors CSC proliferation was suppressed.

The effects of PI3K/mTOR inhibition in combination with differentiating agents on cell migration. GBM cells treated with RAPA (100 $\mathrm{nM})$, LY $(10 \mu \mathrm{M})$ and ATRA $(10 \mu \mathrm{M})$ as single agents all demonstrated decreased cellular motility as compared with control. Among these agents, LY exhibited the highest degree of suppression. Treatment with LY and ATRA in combination had a greater suppressive effect than compared to RAPA and ATRA. Interestingly, the combination of all three agents; LY, RAPA and ATRA, had a synergistic effect causing a complete halt in GBM cell migration (Fig. 4).

\section{Discussion}

The findings of the present study provide further evidence on the targeting of CSCs of GBM by differentiating agent ATRA in combination with $\mathrm{PI} 3 \mathrm{~K} / \mathrm{mTOR}$ inhibitors. The central role of these CSCs in the recurrence and aggressive nature of this disease has made them a key target in its management (5-7). There is abundant evidence of the presence of CSCs within GBM, it has been shown that the expression of stem cell markers (e.g. Nestin and JNK), in tumor and peri-tumor areas can serve as a prognostic indicator (23).

Nestin, a cytoskeletal intermediate filament has long been described as a marker for neural stem cells (24). The confirmation of Nestin expression in GBM neurospheres by immunofluoresence suggests further evidence for the presence of stem cells in GBM. In addition, the loss of Nestin expression following treatment with ATRA is indicative of cellular differentiation occurring in a time-dependent manner. In comparison, upon treatment with the mTORC1 inhibitor RAPA, there was a marked translocation of Nestin expression from the cytoplasm to the nucleus, suggesting that the cells underwent other molecular alterations over differentiation.

Similar results were seen on western blot analysis with a reduction in Nestin expression following ATRA treatment. The combination of ATRA with PI3k inhibitor LY, mTORC1 inhibitor RAPA, and the MEK1/2 inhibitor U0126 also caused 


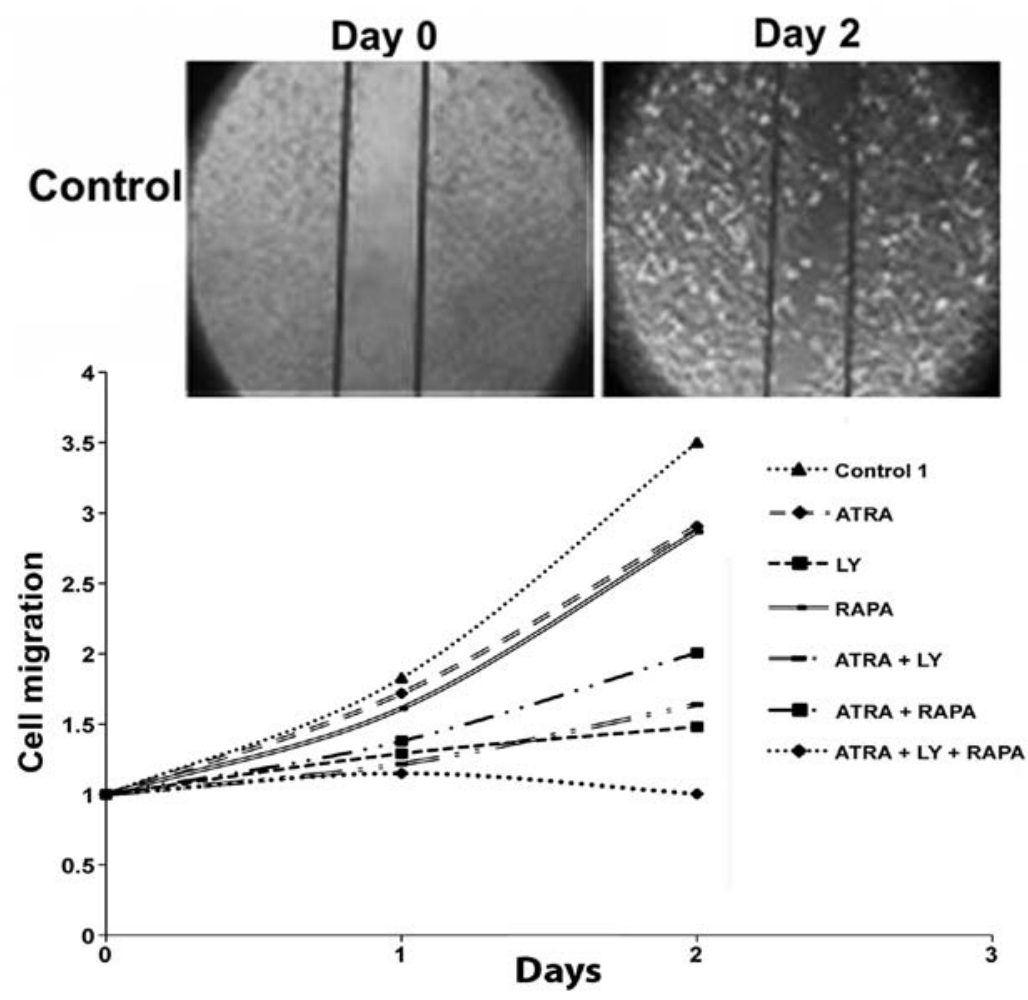

Figure 4. Migration assays for GBM cells. Graphical representation of scratch migration data for GBM cells measured at 1 and 2 days. Control cells show exponential migration. Cells were treated with LY, RAPA, ATRA and various combinations which all had decreased migrations compared to control. The cells treated with the triple combination of LY, RAPA and ATRA had a significantly greater decrease in migration, suggesting a synergistic effect of PI3K inhibition, mTOR inhibition and differentiating agent on GBM cell migration.

a suppression of Nestin. When investigating the mechanism underlying this phenomenon it became suggestive that ATRAinduced differentiation was mediated by the activation of ERK1/2 (pERK1/2). This was demonstrated by the fact that after treatment of cells with ATRA an increase in pERK1/2 expression was observed (Fig. 2B). ERK1/2 is a downstream signaling molecule of the Ras-Raf-MEK1/2 signaling pathway that has been found implicated in many cancers including GBM $(10,25)$. Studies have also shown that MEK1/2, ERK1/2, and mTOR pathways are involved in a mutual negative feedback loop mediated via p70-S6K, in which each pathway can inhibit or activate the other affecting cellular proliferation or differentiation $(26,27)$. This has also led to previous findings by our group that GBM CSCs treated with both MEK1/2, ERK1/2 inhibitors and PI3K/mTOR inhibitors are more effective than either inhibition alone (10). Alternatively ERK1/2 inactivation with U0126 promoted differentiation while inhibiting proliferation (28). Our findings suggest that ATRA results in differentiation through the activation of ERK1/2, and that this effect can be prevented with U0126 treatment. These observations have pertinent clinical relevance since temozolamide resistance in GBM has been found to be mediated by dysregulation of MEK1/2, and ERK1/2 pathways and the inhibition of ERK1/2 pathway may sensitize cells to chemotherapy treatment (29). One notable factor in our results is that treatment with ATRA and U0126 in combination reversed the activation of ERK 1/2 resulting from ATRA treatment alone, the combined treatment did not completely halt differentiation as there was still a significant decrease in Nestin expression with combination treatment. This suggests the possibility of other mechanisms by which ATRA is able to induce differentiation.

Other important results of this investigation include a functional assessment of CSCs. Our results confirm previous findings that inhibition of mTOR or PI3K leads to the reduction of cellular proliferation and migration (30). We have confirmed a similar effect on cell proliferation in GBM CSC populations by demonstrating that mTORC1 inhibitor RAPA, and PI3K inhibitor LY suppressed neurosphere growth, independent of the use of ATRA. The lack of additional suppression in the presence of inhibitors suggests that the dose and time of treatment was optimally achieved, and further decline in proliferation with the addition of secondary agents was unlikely. In addition, we examined the effect of PI3K/ mTOR inhibition on GBM cellular migration. We illustrated that combination of PI3K inhibition, mTOR inhibition and treatment with differentiating agent ATRA had a synergistic effect resulting in the least amount of cellular migration. This may be mediated through mechanisms involving the balance between ATRA-induced differentiation and PI3K/mTOR inhibition regulating proliferation and migration.

CSC proliferation and migration have important clinical implications because these properties relate directly to the invasive nature of GBM. Thus, evidence that inhibitors of mTOR and differentiating agents can negatively affect proliferation and migration suggest their potential therapeutic use. The finding that the use of a differentiating agent in combination with mTOR pathway inhibitors had a synergistic effect in preventing cell migration is of particular significance. This indicates the possibility of targeting CSCs by inducing 
differentiation, followed by treatment with mTOR inhibitors. This is echoed by clinical findings in other cancer types that are already examining inhibition of ERK1/2 and mTOR pathways using existing inhibitors. A phase I trial looked at 236 patients with advanced colorectal cancer treated with a PI3K inhibitor, a MAPK inhibitor, or a combination of the two (31). The results illustrated that dual inhibition was superior in efficacy as compared to inhibition of a single pathway. Therefore, elucidating the mechanism of only a marginal benefit in an early phase trial of mTOR inhibitors (temsirolimus) alone as treatment modality for recurrent GBM (32).

In conclusion, this investigation demonstrates that ATRAinduced differentiation is another mechanism of targeting the MAPK/ERK1/2 pathway, and that treatment of cells with ATRA in combination with PI3K and/or mTOR inhibition was an effective strategy for decreasing CSC proliferation and migration. Thus, using differentiating agents along with inhibitors of mTOR pathways provides a molecular basis for the treatment of GBM.

\section{Acknowledgements}

We gratefully acknowledge the American Research Foundation for their support, and the work done by Michael Pendergast in the administration and maintenance of the laboratory.

\section{References}

1. Lawrence YR, Mishra MV, Werner-Wasik M, Andrews DW, Showalter TN, Glass J, et al: Improving prognosis of glioblastoma in the 21st century: who has benefited most? Cancer 118: 4228-4234, 2012.

2. Wiedemeyer R, Brennan C, Heffernan TP, Xiao Y, Mahoney J, Protopopov A, et al: Feedback circuit among INK4 tumor suppressors constrains human glioblastoma development. Cancer Cell 13: 355-364, 2008.

3. Ohgaki $\mathrm{H}$ and Kleihues P: Genetic pathways to primary and secondary glioblastoma. Am J Pathol 170: 1445-1453, 2007.

4. Endersby R and Baker SJ: PTEN signaling in brain: neuropathology and tumorigenesis. Oncogene 27: 5416-5430, 2008.

5. Holland EC, Hively WP, DePinho RA and Varmus HE: A constitutively active epidermal growth factor receptor cooperates with disruption of G1 cell-cycle arrest pathways to induce glioma-like lesions in mice. Genes Dev 12: 3675-3685, 1998.

6. Galli R, Binda E, Orfanelli U, Cipelletti B, Gritti A, De Vitis S, et al: Isolation and characterization of tumorigenic, stem-like neural precursors from human glioblastoma. Cancer Res 64: 7011-7021, 2004

7. Sanai N, Alvarez-Buylla A and Berger MS: Neural stem cells and the origin of gliomas. N Engl J Med 353: 811-822, 2005.

8. Singh SK, Hawkins C, Clarke ID, Squire JA, Bayani J, Hide T, et al: Identification of human brain tumour initiating cells. Nature 432: 396-401, 2004

9. Ignatova TN, Kukekov VG, Laywell ED, Suslov ON, Vrionis FD and Steindler DA: Human cortical glial tumors contain neural stem-like cells expressing astroglial and neuronal markers in vitro. Glia 39: 193-206, 2002.

10. Jhanwar-Uniyal M, Albert L, McKenna E, Karsy M, Rajdev P, Braun A and Murali R: Deciphering the signaling pathways of cancer stem cells of glioblastoma multiforme: role of Akt $/ \mathrm{mTOR}$ and MAPK pathways. Adv Enzyme Regul 51: 164-170, 2011.

11. Manning BD and Cantley LC: AKT/PKB signaling: navigating downstream. Cell 129: 1261-1274, 2007.

12. Jhanwar-Uniyal M, Jeevan D, Neil J, Shannon C, Albert L and Murali R: Deconstructing mTOR complexes in regulation of glioblastoma multiforme and its stem cells. Adv Biol Regul 53: 202-210, 2013

13. Sarbassov DD, Ali SM and Sabatini DM: Growing roles for the mTOR pathway. Curr Opin Cell Biol 17: 596-603, 2005.
14. Gan B, Sahin E, Jiang S, Sanchez-Aguilera A, Scott KL, Chin L, et al: mTORC1-dependent and -independent regulation of stem cell renewal, differentiation, and mobilization. Proc Natl Acad Sci USA 105: 19384-19389, 2008.

15. Das A, Banik NL and Ray SK: Retinoids induced astrocytic differentiation with down regulation of telomerase activity and enhanced sensitivity to taxol for apoptosis in human glioblastoma T98G and U87MG cells. J Neurooncol 87: 9-22, 2008.

16. Guan K, Chang H, Rolletschek A and Wobus AM: Embryonic stem cell-derived neurogenesis. Retinoic acid induction and lineage selection of neuronal cells. Cell Tissue Res 305: 171-176, 2001.

17. Das A, Banik NL and Ray SK: Molecular mechanisms of the combination of retinoid and interferon-gamma for inducing differentiation and increasing apoptosis in human glioblastoma T98G and U87MG cells. Neurochem Res 34: 87-101, 2009.

18. Zhang R, Banik NL and Ray SK: Combination of all-trans retinoic acid and interferon-gamma upregulated $\mathrm{p} 27^{(\mathrm{kipl})}$ and down regulated CDK2 to cause cell cycle arrest leading to differentiation and apoptosis in human glioblastoma LN18 (ptenproficient) and U87MG (pten-deficient) cells. Cancer Chemother Pharmacol 62: 407-416, 2008.

19. Haque A, Das A, Hajiaghamohseni LM, Younger A, Banik NL and Ray SK: Induction of apoptosis and immune response by all-trans retinoic acid plus interferon-gamma in human malignant glioblastoma T98G and U87MG cells. Cancer Immunol Immunother 56: 615-625, 2007.

20. Defer GL, Adle-Biassette H, Ricolfi F, Martin L, Authier FJ, Chomienne $\mathrm{C}$, et al: All-trans retinoic acid in relapsing malignant gliomas: clinical and radiological stabilization associated with the appearance of intratumoral calcifications. J Neurooncol 34: 169-177, 1997.

21. Clark MJ, Homer N, O'Connor BD, Chen Z, Eskin A, Lee H, et al: U87MG decoded: the genomic sequence of a cytogenetically aberrant human cancer cell line. PLoS Genet 6: e1000832, 2010.

22. Diserens AC, de Tribolet N, Martin-Achard A, Gaide AC, Schnegg JF and Carrel S: Characterization of an established human malignant glioma cell line: LN-18. Acta Neuropathol 53: 21-28, 1981

23. Mangiola A, Lama G, Giannitelli C, De Bonis P, Anile C, Lauriola L, et al: Stem cell marker nestin and c-jun NH2-terminal kinases in tumor and peritumor areas of glioblastoma multiforme: possible prognostic implications. Clin Cancer Res 13: 6970-6977, 2007.

24. Lendahl U, Zimmerman LB and McKay RD: CNS stem cells express a new class of intermediate filament protein. Cell 60: 585-595, 1990 .

25. Roskoski R: ERK1/2 MAP kinases: structure, function, and regulation. Pharmacol Res 66: 105-143, 2012.

26. Albert L, Karsy M, Murali R and Jhanwar-Uniyal M: Inhibition of mTOR activates the MAPK pathway in glioblastoma multiforme. Cancer Genomics Proteomics 6: 255-261, 2009.

27. Sunayama J, Matsuda K, Sato A, Tachibana K, Suzuki K, Narita $\mathrm{Y}$, et al: Crosstalk between the PI3K/mTOR and MEK/ERK pathways involved in the maintenance of self-renewal and tumorigenicity of glioblastoma stem-like cells. Stem Cells 28: 1930-1939, 2010

28. Wang B, Gao Y, Xiao Z, Chen B, Han J, Zhang J, et al: Erk1/2 promotes proliferation and inhibits neuronal differentiation of neural stem cells. Neurosci Lett 461: 252-257, 2009.

29. Sato A, Sunayama J, Matsuda K, Seino S, Suzuki K, Watanabe E, et al: MEK-ERK signaling dictates DNA-repair gene MGMT expression and temozolomide resistance of stem-like glioblastoma cells via the MDM2-p53 axis. Stem Cells 29: 1942-1951, 2011.

30. Gulati N, Karsy M, Albert L, Murali R and Jhanwar-Uniyal M: Involvement of mTORC1 and mTORC2 in regulation of glioblastoma multiforme growth and motility. Int J Oncol 35: 731-740, 2009.

31. Shimizu T, Tolcher AW, Papadopoulos KP, Beeram M, Rasco DW, Smith LS, et al: The clinical effect of the dual-targeting strategy involving PI3K/AKT/mTOR and RAS/MEK/ERK pathways in patients with advanced cancer. Clin Cancer Res 18: 2316-2325, 2012.

32. Galanis E, Buckner JC, Maurer MJ, Kreisberg JI, Ballman K, Boni J, et al: Phase II trial of temsirolimus (CCI-779) in recurrent glioblastoma multiforme: a north central cancer treatment group study. J Clin Oncol 23: 5294-5304, 2005. 